\title{
Editorial
}

\section{Biomarkers in Public Health}

Every disease has preclinical symptoms. For example, rising cholesterol is indicative of impending cardiovascular indications, high blood pressure an indicator for stroke, and high level of sugar for Type II diabetes. These preclinical symptoms are considered to be risk factors and are usually treated in order to reduce the risk. However, not everyone with these risk factors develop disease. Detection of a disease based on associated preclinical symptoms leads to overdiagnosis and overtreatment. Screening of cancer offers a good example of this phenomenon. It is estimated that about $25 \%$ of mammography-detected breast cancers, $50 \%$ of chest X-ray and/or sputum-detected lung cancers, and $60 \%$ of prostate-specific antigen (PSA)-detected prostate cancers are overdiagnosed. For those readers who are not familiar with these terms, the simple definition of overdiagnosis is when a condition is diagnosed that would not cause symptoms or death. However overdiagnosis should not be confused with falsepositive cases based on a screen-positive test in an individual who is subsequently found not to have cancer. In contrast, an overdiagnosed patient has a tumor that possesses pathological criteria for cancer.

In many countries health care spending is rising faster than overall economic growth, so almost all countries have seen health care spending increase as a percentage of their gross domestic product (GDP) over time. In the United States, which has both a high level of health spending per capita and a relatively high rate of real growth in spending, the share of GDP devoted to health care spending grew from 9\% of GDP in 1980 to $16 \%$ of GDP by 2008 . The dramatic rise in health care costs in the past three decades is partly related to the proliferation of new technologies. The US Congressional Budget Office projected that the total spending on health would rise from $16 \%$ of GDP in 2007 to 25 percent in 2025, 37 percent in 2050 and 40 percent in 2082. More recent analysis indicates that technological change such as new procedures, products and capabilities, is the primary explanation of the historical increase in expenditure. Excessive diagnostic evaluations with body scans or blood tests will always find something amiss. The US Office of Management and Budget estimates they contribute to the estimated $\$ 700$ billion in wasted annual health costs.

To address this problem, we must take advantage of increased knowledge of disease causation and factors affecting the biological initiation and progression of the disease. The last three decades have witnessed a rapid advancement and diffusion of technology in health services. Technological innovations have given health service providers the means to diagnose and treat an increasing number of illnesses, including cancer, with greater specificity and precision. In this effort, research on biomarkers for disease detection and risk assessment has taken a center stage. For the first time, scientists have the molecular technologies to decipher and understand these biomarkers and to apply them to earlier detection. For example, by identifying people at high risk of developing cancer, it would be possible to develop intervention efforts on prevention rather than treatment.

Once fully developed and validated, the regular clinical use of biomarkers in early detection and risk assessment will meet nationally recognized health care needs: detection of cancer at its earliest stage. Biomarkers are biological molecules found in blood, bodily fluids, tissues or the tumor itself. Biomarkers can be a sign of a normal/abnormal process, or of disease. Biomarkers are said to play an important role in personalized medicine, i.e., personalized medicine is the customization of treatment tailored to the individual patient by use of genetic or other information. Unlike traditional management of disease "one size fits all" in which treatment is based on the individual patient's clinical signs and symptoms, medical and family history, and data from laboratory and imaging evaluation to diagnose. Personalized medicine is aimed at tailoring drug therapy specific to certain molecular genes or proteins at a dosage that is most appropriate for an individual 
patient, with the potential benefits of increasing the efficacy and safety of medications. Personalized medicine uses biological "biomarkers" - such as DNA sequences or the presence or absence of drug receptors - as an indicator of how patients should be treated, as well as to estimate the likelihood that the intervention will be effective. For example, successful personalized treatment is being administered to breast, colon and lung cancer patients based on the measurements of erbB2 and EGFR before selecting proper treatments.

While biomarkers could be a boon to personalized medicine through precise diagnosis and treatment, it is not without challenges. Use of poorly designed biomarker-based tests could lead to overdiagnosis and treatment. Therefore, there must be adequate information on the nature and the magnitude of the trade-off between overdiagnosis and treatment. Researchers need to develop better estimates of the magnitude of overdiagnosis and develop clinical strategies to help mini- mize it. However, biomarkers for earlier detection and risk assessment have not achieved the level of confidence required for clinical applications. It is hoped that diagnostic technologies, proteomics and genomics, will lead to the development of disease-specific biomarkers, which could reduce the health care cost by providing noninvasive, sensitive and reliable assays at a fraction of the cost.

Several articles in this issue of the journal address a number of biomarkers for early detection, diagnosis, and prognosis. Although these biomarkers must be fully validated for their clinical applications using appropriate samples, study designs, and the appropriate patient populations, these publications are a testimonial to the rapid progress made in understanding the biology of disease.

Sudhir Srivastava

Editor-in-Chief 